\title{
The relationship of vibrational energy distribution on the level of driver detection
}

\author{
SABARIAH MOHD YUSOFF ${ }^{1,2}$, JOSEPH GIACOMIN ${ }^{2}$ \\ ${ }^{1}$ Quality Engineering Section, University Kuala Lumpur, Malaysian Institute of Industrial \\ Technology, 81750 Bandar Seri Alam, Johor, Malaysia \\ ${ }^{2}$ Human Centred Design Institute, Brunel University, Uxbridge, UB8 3PH, UK
}

\begin{abstract}
A laboratory-based experiment was conducted to measure the effect of vibrational energy distribution on human cognitive detection of road surface based on steering wheel vibration. The test stimuli used in the current study were ten steering wheel acceleration time histories of mid-sized European automobiles. The ten original steering wheel time histories were manipulated via digital Butterworth filters to eliminate four different frequency bands from the steering wheel vibration spectrum of within 20 to $60 \mathrm{~Hz}$. The ensemble, composed of both the original and the manipulated time histories, was used to perform a laboratory-based detection. During the test, participants were asked to judge if the actuated acceleration stimulus transmitted came from the road surface shown on photographs featured on a board directly in front of the test bench and rate the confidence of their judgement on a five-point scale $((1)=$ very sure there was no signal $-(5)=$ very sure there was a signal)). The findings suggest that the elimination of vibrational energy in the frequency band of 26.32 to $34.64 \mathrm{~Hz}$ can be highly detrimental to human cognitive detection of road surface types and compromise the steering wheel feedback the most. The elimination can lead to the correct detection of road surfaces.
\end{abstract}

\section{Introduction}

Steer-by-wire systems have become a central issue in the automobile industry. With such systems there is often an objective to minimise vibrations on the steering wheel to increase driver comfort. Nevertheless, steering wheel vibration is also recognised as an important medium that assists drivers in judging the vehicle, road and to indicate important information such as the presence of danger as well as the vehicle's subsystems dynamics. This has led to studies of the possible role of vibrational stimuli towards informing drivers of environment conditions such as road surface type. Numerous prior studies were done to identify how characteristics of steering wheel vibrational stimuli might influence human cognitive detection of road surfaces, beginning with primary characteristics of the vibrational stimuli (scale and bandwidth) by [1], [2] and [3]. The results from scaling and bandwidth experiments suggested that a single, optimal, acceleration gain does not exist and steering wheel acceleration frequencies exceeding $60 \mathrm{~Hz}$ were used by the human for purposes of road surface type detection.

In [4], further investigation of the effect of vibrational energy distributions has been done. They highlighted that the elimination of vibrational energy within the frequency band of 20 to $60 \mathrm{~Hz}$ is highly detrimental to the task of detecting road surface. Even though frequency band of 20 to $60 \mathrm{~Hz}$ was found to be a significant impact on the drivers' awareness, the frequency band was known to contain resonant behaviours of numerous chassis and steering systems [5]. The steering wheel feedback can hence be determined by reducing the range of the eliminated frequency band at 20 to $60 \mathrm{~Hz}$. Therefore, the current study aimed to measure the effect of vibrational energy distribution within the frequency band of 20 to 
$60 \mathrm{~Hz}$ on the human cognitive detection of road surface types and to identify the interval frequency band that most compromised with steering feedback.

\section{Theory of Signal Detection with Rating Scale}

Previous studies evaluated human detection response using binary response procedure [1], [2], [3] and [4]. However, an alternative procedure involving detection using a continuum might prove beneficial towards improving its accuracy and resolution [6]. Consequently, the Theory of Signal Detection (TSD) with a rating scale was applied in this study.

TSD facilitates the measurements and quantification of how people actually behave in detection situations based on statistical decision theory [7]. TSD is applicable in any situation where a signal is detected in the presence of background noise. Figure 1 presents graphically the distribution curve in theory of signal detection.

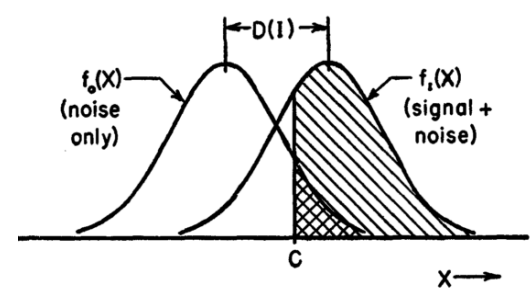

Figure 1. Noise and signal plus noise distributions [8].

During the signal detection task, the observer must make an observation $(x)$ and then make a decision about the observation. In each trial, the observer must decide whether $x$ is due to a signal added to the background noise or to the noise alone. In rating scale procedure, the assumption is that the observer would say 'Yes' where they report a signal was almost certainly present. Accordingly, every trial the observer used in the first category is treated as if the he/she had said 'Yes', while all others where the remaining categories were used are treated as if the observer had said 'No'. Analysis of the signal $(s)$ and noise $(n)$ trials in this manner had allowed researchers to estimate the hit rate, $\mathrm{P}(\mathrm{S} \mid s)$ and false alarm, $\mathrm{P}(\mathrm{S} \mid n)$ respectively [7].

Table 1 shows the stimulus-response matrix for the rating procedure containing the sequence of two physical stimulus events $(s$ and $n$ ) and the five scale responses. The first scale represents $s$ as almost certainly not present, while the fifth as almost certainly present. The scale between one and five represents lesser degree of certainty about the occurrence or non-occurrence of $s$. Meanwhile, Table 2 displays the reduction of the rating procedure matrix to a binary response procedure matrix used in the analysis. The details were described in [7].

Table 1. Stimulus-response matrix for rating procedure.

\begin{tabular}{|c|c|c|c|c|c|}
\hline & \multicolumn{5}{|c|}{ Rating Response } \\
\hline & 1 & 2 & 3 & 4 & 5 \\
\hline \multirow{2}{*}{ 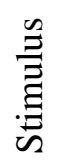 } & $\mathrm{P}(1 \mid s)$ & $\mathrm{P}(2 \mid s)$ & $\mathrm{P}(3 \mid s)$ & $\mathrm{P}(4 \mid s)$ & $\mathrm{P}(5 \mid s)$ \\
\hline & $\mathrm{P}(1 \mid n)$ & $\mathrm{P}(2 \mid n)$ & $\mathrm{P}(3 \mid n)$ & $\mathrm{P}(4 \mid n)$ & $\mathrm{P}(5 \mid n)$ \\
\hline
\end{tabular}


Table 2. Reduction of matrix from rating to binary procedure.

\begin{tabular}{|c|c|c|}
\cline { 2 - 3 } \multicolumn{1}{c|}{} & \multicolumn{2}{|c|}{ Presumed Binary Response } \\
\cline { 2 - 3 } \multicolumn{1}{c|}{} & $\mathbf{S}$ & $\mathbf{N}$ \\
\hline \multirow{2}{*}{} & $\mathrm{Pit}$ & Miss \\
\cline { 2 - 3 } & $\mathrm{P}(\mathrm{S} \mid s)=\mathrm{P}(4 \mid s)+\mathrm{P}(5 \mid s)$ & Correct rejection \\
\cline { 2 - 3 } & $\mathrm{P}(\mathrm{S} \mid n)=\mathrm{P}(1 \mid n)+\mathrm{P}(2 \mid n)+\mathrm{P}(3 \mid n)$ & \\
\hline
\end{tabular}

\section{Experiment Road Surface Detection}

\subsection{Test Facility}

The study employed steering wheel rotational vibration test rig to perform laboratory experiments as shown in Figure 2. The schematic diagram and geometric dimensions of the test rig were described in detail by [9].

An evaluation was done to determine the stimuli reproduction accuracy of the test rig facility. The accuracy of the target stimuli reproduction was quantified by measuring the r.m.s. difference between the actuated signal and the target signal. Seven participants were used in the pre-test process to also consider the possible differences in bench response caused by the impedance loading discrepancies on the steering wheel of people with different sizes. The pre-test results suggested that the maximum rate of error between the level of r.m.s. acceleration's target signal and the actuated signal was found to be less than $5 \%$ for all stimuli used.

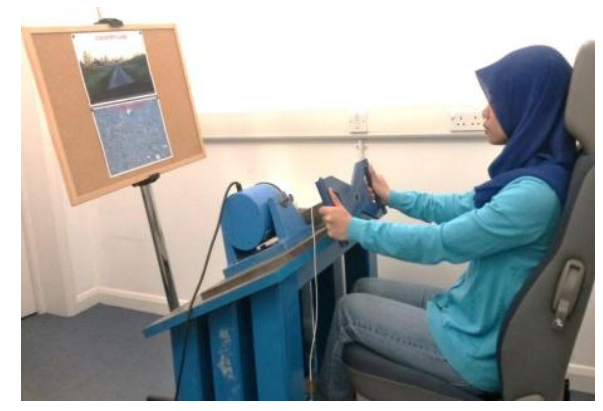

Figure 2. Steering wheel test rig.

\subsection{Test Stimuli}

The test stimuli used were ten steering wheel acceleration time histories selected from an extensive database of previous road test measurements done by the Perception Enhancement Research Group of Brunel University, London [4] and [9]. The steering wheel vibration stimuli were chosen on the premise that steering wheel vibration is caused mainly by the act of driving over a road surface. This decision was made based on the results of a previous questionnaire-based study [10] which suggested that respondents considered steering wheel vibration to be particularly useful in the task of detecting road surface types.

The ten steering wheel time histories with their global statistics properties are listed in Table 3. This is followed by the data of the aerial and distant view of the ten base stimuli while driving as well as the original acceleration time histories extracted from the 10-second segment in Figure 3. 
Table 3. Global statistical properties of the steering wheel acceleration time histories used for the laboratory test stimuli.

\begin{tabular}{|c|c|c|c|c|c|}
\hline $\begin{array}{c}\text { Type of } \\
\text { Road Surface }\end{array}$ & $\begin{array}{l}r . m . s \\
\left(\mathbf{m} / \mathbf{s}^{2}\right)\end{array}$ & $\begin{array}{l}\text { Vehicle speed } \\
(\mathrm{km} / \mathrm{h})\end{array}$ & $\begin{array}{c}\text { Kurtosis } \\
\text { (Dimensionless) }\end{array}$ & $\begin{array}{c}\text { Skewness } \\
\text { (Dimensionless) }\end{array}$ & $\begin{array}{c}\text { Crest Factor } \\
\text { (Dimensionless) }\end{array}$ \\
\hline Broken* & 1.317 & 40 & 3.484 & -0.051 & 3.838 \\
\hline Broken Concrete & 1.733 & 50 & 3.214 & 0.010 & 3.358 \\
\hline Broken Lane* & 1.784 & 50 & 3.631 & -0.028 & 4.399 \\
\hline Cobblestone* & 1.310 & 30 & 3.163 & 0.007 & 3.893 \\
\hline Country Lane ${ }^{*}$ & 2.048 & 40 & 3.271 & -0.066 & 3.487 \\
\hline Concrete ${ }^{*}$ & 0.094 & 96 & 3.079 & 0.049 & 3.467 \\
\hline Harsh & 1.265 & 40 & 18.831 & 1.142 & 7.279 \\
\hline Low Bump & 0.139 & 50 & 6.426 & 0.329 & 5.350 \\
\hline Noise & 0.746 & 80 & 2.928 & 0.088 & 3.464 \\
\hline Tarmac $^{*}$ & 0.055 & 96 & 3.005 & 0.066 & 3.639 \\
\hline
\end{tabular}

* Classified as mildly non-stationary signals [5].

For each of the ten original steering wheel time histories ( 3 to $125 \mathrm{~Hz}$ ), four different frequency bands within 20 to $60 \mathrm{~Hz}$ were eliminated. To differentiate the test stimuli $f_{\text {test }}$ from the frequency of reference stimuli $f_{r e f}$, the study used the Weber fractions $\left(J N D F / f_{r e f}\right)$ calculation to determine the four different frequency bands, within the interval from 20 to $60 \mathrm{~Hz}$, to be eliminated. The variable increment was fixed by $f_{\text {test }}=f_{\text {ref }}+\Delta f$ to differentiate the test stimuli $f_{\text {test }}$ from the frequency of reference stimuli $f_{\text {ref }}[11]$.

$$
J N D F=\frac{\Delta f}{f}
$$

In [12] suggested that vibration intensity needs to be reduced by at least $10 \%$ for the changes to be detected by a person; hence Equation 1 can be simplified to:

$$
J N D F=\frac{f_{n+1}-f_{n}}{0.1 f_{n}}, n=1,2,3,4
$$

where $n$ is the total number of the frequency bands that were eliminated. Since $f_{1}=20$ and $f_{4}=60$, the value for frequency band intervals was found to be equal on a logarithmic scale of 3.161 by simplifying Equation 2. After all the values were substituted, the general formula to determine the frequency band intervals were identified as follows:

$$
f_{n}=20(1.3161)^{n-1}, n=1,2,3,4,5
$$

From Equation 3, the frequency bands to be eliminated were identified as 20 to $26.32 \mathrm{~Hz}, 26.32$ to $34.64 \mathrm{~Hz}, 34.64$ to $45.59 \mathrm{~Hz}$ and 45.59 to $60 \mathrm{~Hz}$, respectively. The elimination for each of the frequency bands involved high-pass filtered and band-pass filtered processes. This was achieved with digital Butterworth filters constructed using the $\mathrm{LMS}^{\circledR}$ TMON software [13].

Figure 4 presents the example of the power spectral density (PSD) data of the original and manipulated high-pass filtered and band-pass filtered for Country Lane, which recorded the highest energy level among the ten road surface studied. The r.m.s. acceleration value $\left(\mathrm{m} / \mathrm{s}^{2}\right)$ for each of test stimuli obtained after the elimination of the chosen frequency bands are shown in Table 4. 

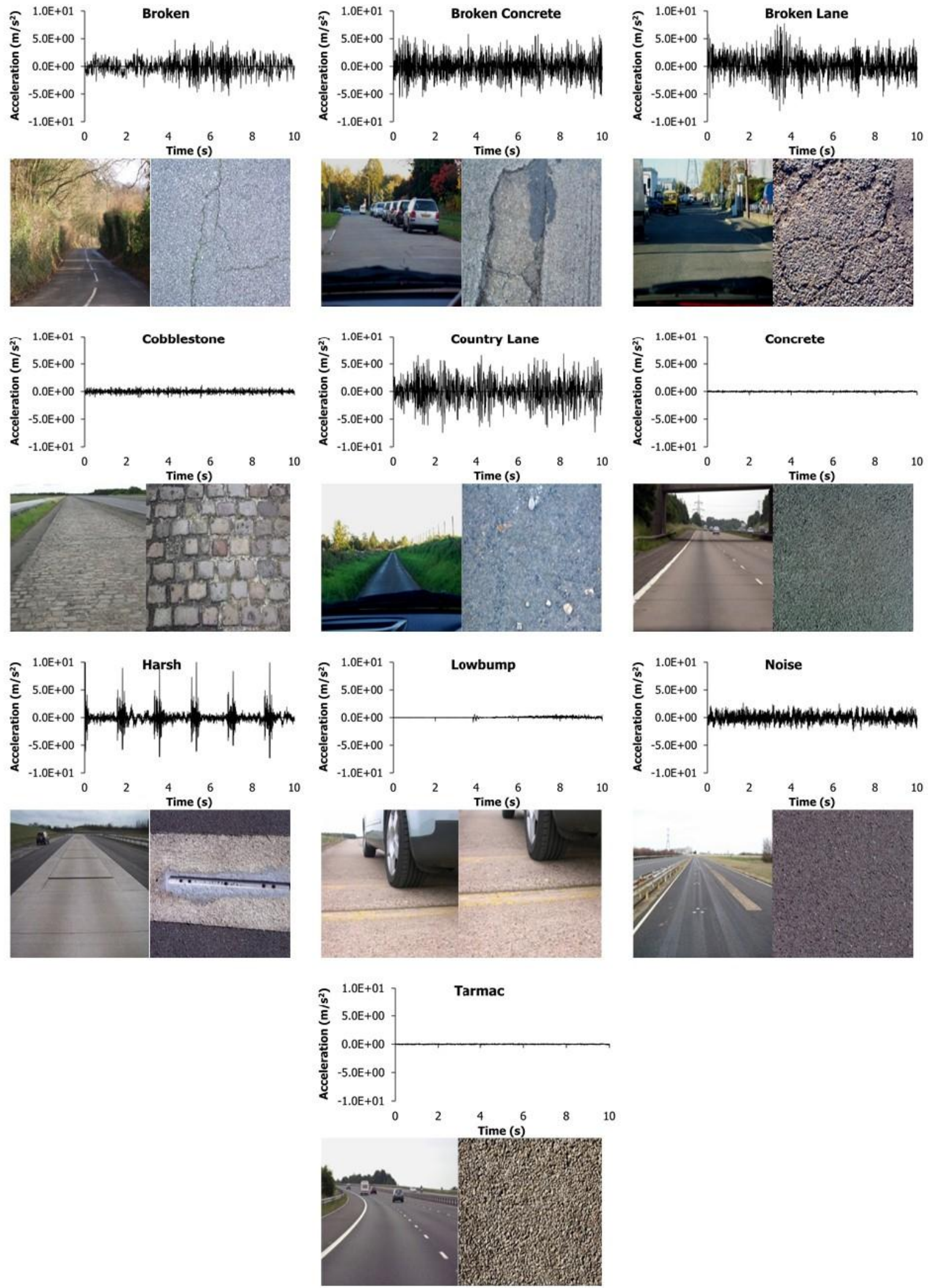

Figure 3. Road surfaces and time history segments extracted from the road test recordings for laboratory stimuli. 


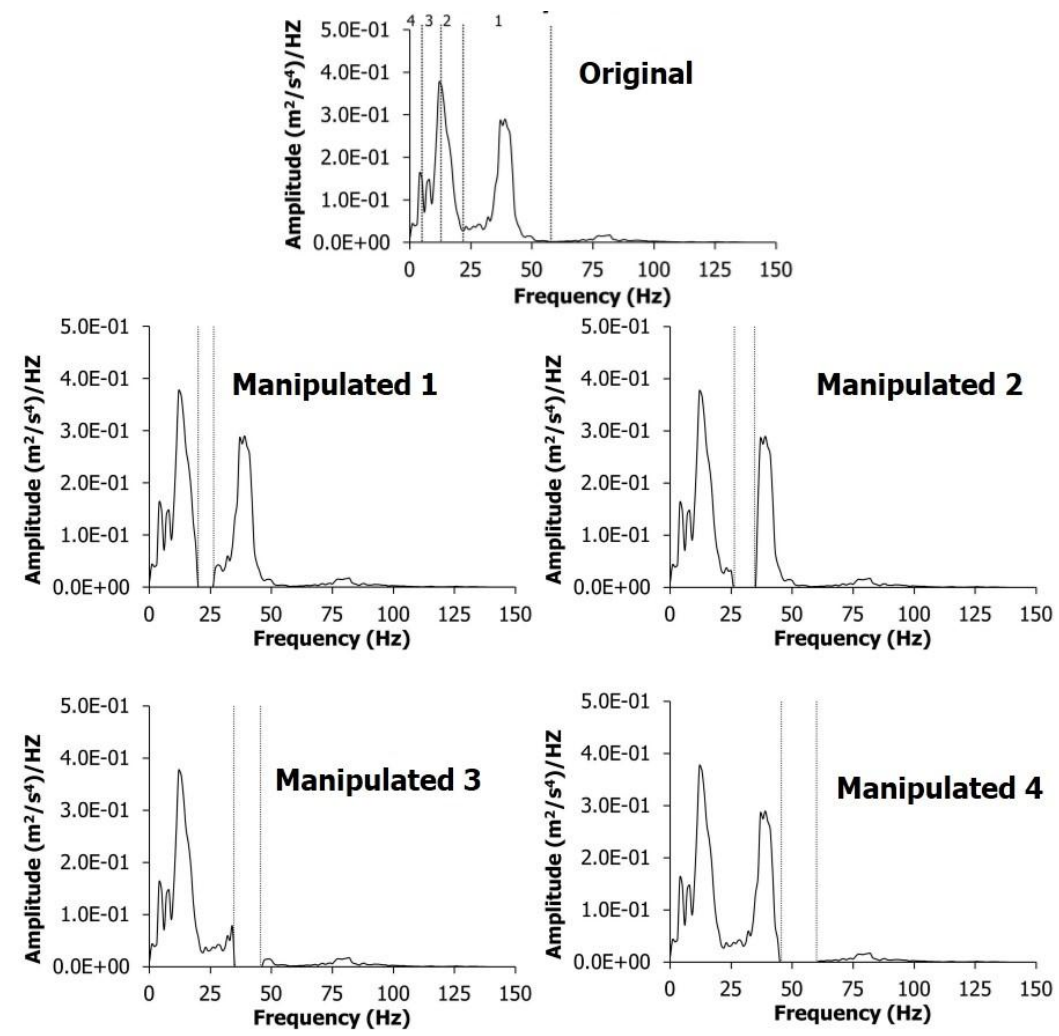

Figure 4. PSD of Country Lane for original and manipulated Butterworth filter used for producing the laboratory test stimuli.

Table 4. The r.m.s. values $\left(\mathrm{m} / \mathrm{s}^{2}\right)$ for original and manipulated Butterworth filter used for producing the laboratory test stimuli.

\begin{tabular}{|c|c|c|c|c|c|}
\hline \multirow{2}{*}{$\begin{array}{c}\text { Type of } \\
\text { Road Surface }\end{array}$} & \multirow{2}{*}{ Original } & \multicolumn{4}{|c|}{ Eliminated Frequency Bands } \\
\hline & & $20-26.32 \mathrm{~Hz}$ & $26.32-34.64 \mathrm{~Hz}$ & $34.64-45.59 \mathrm{~Hz}$ & $45.59-60 \mathrm{~Hz}$ \\
\hline Broken & 1.317 & 0.764 & 0.726 & 1.275 & 1.306 \\
\hline Broken Concrete & 1.733 & 1.519 & 1.464 & 1.157 & 1.680 \\
\hline Broken Lane & 1.784 & 1.644 & 1.693 & 1.415 & 1.720 \\
\hline Cobblestone & 1.310 & 1.092 & 1.196 & 1.212 & 1.281 \\
\hline Country Lane & 2.048 & 1.853 & 1.888 & 1.623 & 2.027 \\
\hline Concrete & 0.094 & 0.081 & 0.088 & 0.089 & 0.089 \\
\hline Harsh & 1.265 & 0.886 & 0.961 & 0.668 & 1.038 \\
\hline Low Bump & 0.139 & 0.091 & 0.116 & 0.128 & 0.136 \\
\hline Noise & 0.746 & 0.646 & 0.676 & 0.589 & 0.680 \\
\hline Tarmac & 0.055 & 0.037 & 0.047 & 0.051 & 0.052 \\
\hline
\end{tabular}

\subsection{Test Subject}

A non-probability purposive sampling strategy [14] was used, where driving experience of a minimum of two years was selected as a primary characteristic and controlled parameter to minimise errors during detection task [15]. Meanwhile, gender and physical body mass were not controlled since there were no significant differences between both parameters in the subjective experience of hand-arm vibration [16], [17] and [18].

Sixteen $(n=16)$ participants took part in the study. Table 5 summarises the controlled and uncontrolled parameters of participants. It is essential to note that none of the participants declared any conditions that might affect their perception of hand-arm vibration. 
Table 5. Anthropometrics and driving experience of test participants.

\begin{tabular}{|c|l|c|c|c|c|}
\hline \multicolumn{2}{|c|}{ Characteristics } & \multicolumn{2}{c|}{ Male (n=8) } & \multicolumn{2}{c|}{ Female (n=8) } \\
\cline { 3 - 6 } \multicolumn{2}{|c|}{} & Mean & SD & Mean & SD \\
\hline \multirow{2}{*}{$\begin{array}{c}\text { Controlled } \\
\text { Parameters }\end{array}$} & Age (years) & 30.88 & 11.95 & 26 & 4.78 \\
\cline { 2 - 6 } & Driving experience (years) & 8.63 & 7.44 & 4.5 & 2.45 \\
\hline $\begin{array}{c}\text { Uncontrolled } \\
\text { Parameters }\end{array}$ & Weight (kg) & 79.75 & 9.88 & 61.69 & 13.25 \\
\cline { 2 - 6 } & Height (m) & 1.77 & 0.10 & 1.62 & 0.04 \\
\hline
\end{tabular}

From Table 5, it is clear that the mean values and standard deviations of the height and mass of the test participants were proximate to the $50^{\text {th }}$ percentile value for the U.K population [19]. The driving experience of participants can be categorised as experienced drivers [20]. A statistical $t$-test performed for the test groups in uncontrolled parameter suggested significant physical differences in height and mass between males and females $(\mathrm{p}<0.05)$.

\subsection{Test Protocol}

Following an email invitation sent to the students' general mailing list of the College of Engineering, Design and Physical Sciences, Brunel University, London, the potential participants were approached by the researcher. Each potential participant was given an information sheet and a consent form describing the purpose, procedures, risks and time commitment entailed in their participation.

Before commencing the test, participants were required to remove any articles of heavy clothing such as coats as well as watches and jewellery. To ensure that the simulated driving postures is as realistic as possible and to minimise the effect of the transmission of vibrations to the hand-arm system [21], each participant were also asked to adjust their seat position and backrest angle and maintain a constant palm grip on the steering wheel with both hands. The room temperature in the laboratory was kept at the range of 20 to $25^{\circ} \mathrm{C}$ to avoid significant environmental effects on participants' skin sensitivities [22]. During the test, each participant were asked to look at a board placed directly in front of the test bench displaying a photograph of the road surface studied (Figure 2). Then, they were asked to judge whether the actuated acceleration stimulus transmitted comes from the road surface shown on photograph. They were also asked to rate the confidence of their judgement on a five-point scale. "One" indicates that they were very sure there was no signal and "two" if they were fairly sure of it. "Three" indicates they were not sure whether there was a signal detected or not. "Four" represents if they were fairly sure there was a signal and "five" if they were very sure of it. Each series of stimuli were separated by a five-second gap to allow participants express their responses towards the stimuli.

Due to the large total numbers of both the stimuli detection (400 detections) and time spent (one and half hours), the experiment design took into account the learning and fatigue effects [23] and [24]. Hence, participants were asked to come on two consecutive days to complete a total of 400 detections consisted of 40 detections for each road surface studied, totalling to 200 detections per day. The order of stimuli arrangements in each series was fully random for each participant.

Each of the ten road surfaces studied consisted of three repetitions of the four high-pass filtered, band-pass filtered and the original base stimulus from the displayed road surface. In addition, a further 25 stimuli were chosen randomly from other stimuli sets of the other nine road surfaces as background noise. Five different series consisting of eight acceleration stimuli were applied to evaluate each road surface types. The duration of each individual test stimulus was ten-seconds.

The facility and the protocol of the experiment was approved by the Ethics Committee of the College of Engineering, Design and Physical Sciences, Brunel University, London. 


\section{Results}

Figure 5 presents the percentage of correct detection as a function of the frequency bands. The line graph shows the relationship between the correct detections of the original and Butterworth filters of steering acceleration stimuli for each of the ten roads studied.
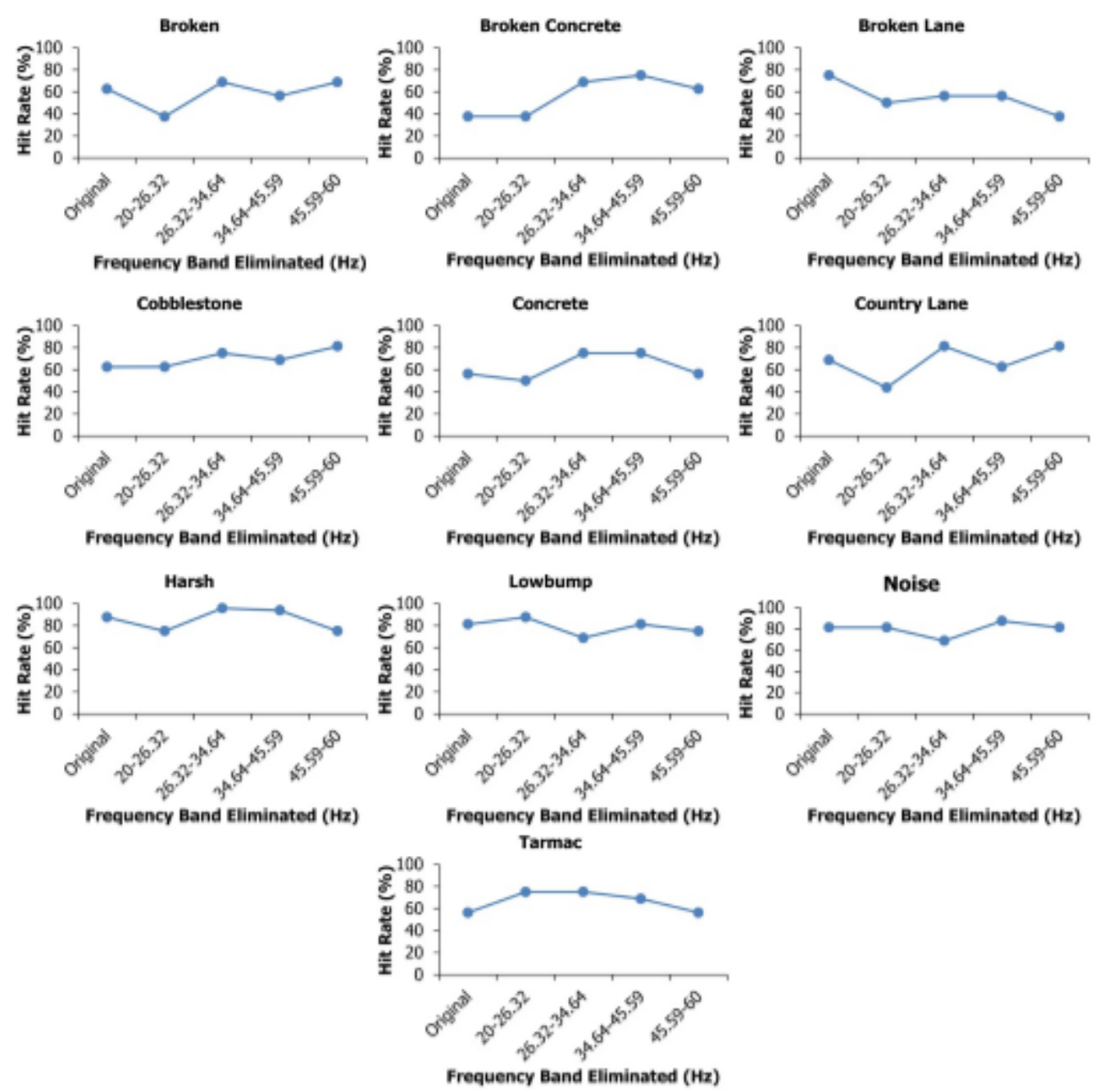

Figure 5. Rate of hit detection for all ten road surfaces studied.

As shown in Figure 5, the percentage of correct detection for the original steering acceleration stimuli for Broken, Broken Lane, Cobblestone, Country Lane, Concrete, Low Bump, Harsh, Noise and Tarmac were higher than 50 percent in each case, with the sole exception of Broken Concrete where the hit rate was 37.6 percent. Qualitatively, the results from the Broken Concrete showed a very different behaviour from that of the other nine test stimuli, suggesting important differences in the underlying energy content.

Next, when the participants were exposed to the frequency band of 20 to $26.32 \mathrm{~Hz}$ which was eliminated from the original time histories. It was found that the percentage of detection had decreased by 12 to 25 percent for Broken, Broken Lane, Country Lane, Concrete and Harsh surface. In contrast, there was a slight increase of 5 to 19 percent for Broken Concrete, Cobblestone, Low Bump, Noise and Tarmac surfaces.

From Figure 5, it can be claimed that the detection ability increased sharply at the frequency band of 26.32 to $34.64 \mathrm{~Hz}$ for all the ten road surfaces, except for Low Bump, Noise and Tarmac, which played an important role in the human's cognitive detection of all the road surfaces studied. 


\section{Discussion}

This study has set out to measure how changes in the vibrational energy within the frequency band of 20 to $60 \mathrm{~Hz}$ may affect human cognitive detection of road surface types based on steering wheel vibration. The results of this study reveal that the detection percentage achieved was less than 100 percent for all ten road surfaces studied. This may be due to the lack of the presence of several key stimuli such as acoustical stimuli. Another possible cause of low level of detection include the signal reproduction of up to $18 \%$ due to the signal distortion, defined as an error phenomenon that causes the appearance of extraneous signals at the output of test equipment [25].

However, the results suggest that the elimination of frequency band 26.32 to $34.64 \mathrm{~Hz}$ made it almost possible for correct detections to be made of the road surfaces and this was observed to be imperative in increasing the sensitivity of human cognitive ability towards detecting conditions of road surfaces. These relationships may be partly explained by the fact that the elimination of the frequency band appeared to have produced the highest peaks of vibrational energy resulting from the resonance in the vehicle dynamics systems such as tires and steering wheel [4]. Moreover, these results are also consistent with those reported by [26], [27] and [5], who suggested that a frequency band of 23 to $58 \mathrm{~Hz}$ is the largest range of frequency that contributes to vehicle dynamics, whereby the band of 20 to $35 \mathrm{~Hz}$ is defined by steering wheel resonance [28].

\section{Conclusion}

This study involved sixteen participants who were exposed to vertical steering wheel vibration stimuli in a laboratory test bench to identify ten different road surfaces. The objective was to establish the most pertinent frequency band, within 20 to $60 \mathrm{~Hz}$ which, if eliminated from the vibrational energy, might affect the level of human detection of road surface types.

The findings suggested that the elimination of vibrational energy in the frequency band of 26.32 to $34.64 \mathrm{~Hz}$ can be highly detrimental to human cognitive detection of road surface types. The findings also demonstrated that the frequency band of 26.32 to 34.64 compromised with the steering wheel feedback the most and the elimination of these frequency band can lead to the correct detection of road surfaces.

\section{References}

[1] Giacomin, J., \& Woo, Y. J., 2005. A study of the human ability to detect road surface type on the basis of steering wheel vibration feedback. Proceedings of the Institution of Mechanical Engineers, Part D: Journal of Automobile Engineering, 219(11), pp. 1259-1270.

[2] Giacomin, J., \& Woo, Y. J., 2006. The role of the scale and the frequency bandwidth of steering wheel vibration on road surface recognition. 8th International Symposium on Advanced Vehicle Control (AVEC '06), Taipei, Taiwan, Aug 20-24th.

[3] Berber-Solano, T. P., \& Giacomin, J. A., 2005. Perception enhancement system for automotive steering. In: Proceedings of the 2005 annual conference on European association of cognitive ergonomics. University of Athens, September. pp. 147-153.

[4] Berber-Solano, T. P., Giacomin, J. A., \& Ajovalasit, M., 2013. Effect of steering wheel acceleration frequency distribution on detection of road type. Ingeniería mecánica, tecnologíay desarrollo, 4(4), pp. 145-151.

[5] Giacomin, J., Steinwolf, A., \& Staszewski, W. J., 2000. Application of mildly nonstationary mission synthesis (MNMS) to automotive road data. Engineering Integrity, 7, pp. 44-56.

[6] Laming, D., 2003. Human judgment: the eye of the beholder. Cengage Learning EMEA.

[7] Green, D. M., \& Swets, J. A., 1966. Signal detection theory and Psychophysics. New York: Wiley.

[8] Fitzhugh, R., 1957. The statistical detection of threshold signals in the retina. The Journal of general physiology, 40(6), pp. 925-948.

[9] Berber-Solano, T. P., Ajovalasit, M., \& Giacomin, J., 2010. Facilitating the driver detection of road surface type by selective manipulation of the steering-wheel acceleration signal. Proceedings of the Institution of Mechanical Engineers, Part D: Journal of Automobile Engineering, 224(10), pp. 1321-1333. 
[10] Gnanasekaran, S., Ajovalasit, M., \& Giacomin, J., 2006. Driver estimation of steering wheel vibration intensity: laboratory-based tests. Journal of the Engineering Integrity Society, 20(1), pp. 25-31.

[11] Bau, O., Poupyrev, I., Israr, A., \& Harrison, C., 2010. TeslaTouch: electrovibration for touch surfaces. In Proceedings of the 23nd annual ACM symposium on User interface software and technology, October, pp. 283-292. ACM.

[12] Morioka, M., \& Griffin, M. J., 2000. Difference thresholds for intensity perception of whole-body vertical vibration: effect of frequency and magnitude. The Journal of the Acoustical Society of America, 107(1), pp. 620-624.

[13] LMS International, 2002. LMS Cada-X Fourier Monitor Manual. Revision 3.5E. Leuvan.

[14] Coolican, H., 1990. Research methods and statistics in psychology. Psychology Press.

[15] Zhao, N., Chen, W., Xuan, Y., Mehler, B., Reimer, B., \& Fu, X., 2014. Drivers' and non-drivers' performance in a change detection task with static driving scenes: is there a benefit of experience?. Ergonomics, 57(7), pp. 998-1007.

[16] Mansfield, N. J., \& Griffin, M. J., 2000. Non-linearities in apparent mass and transmissibility during exposure to whole-body vertical vibration. Journal of biomechanics, 33(8), pp. 933 $-941$.

[17] Neely, G., \& Burström, L., 2006. Gender differences in subjective responses to hand-arm vibration. International journal of industrial ergonomics, 36(2), pp. 135-140.

[18] Jeon, B. H., Ajovalasit, M., \& Giacomin, J., 2009. Effects of gender differences on the subjective perceived intensity of steering wheel rotational vibration based on a multivariate regression model. International Journal of Industrial Ergonomics, 39(5), pp. 736-743.

[19] Pheasant, S., \& Haslegrave, C. M., 2005. Bodyspace: Anthropometry, ergonomics and the design of work. CRC Press.

[20] Mulder, M., Abbink, D., \& Boer, E. R., 2008. The effect of haptic guidance on curve negotiation behavior of young, experienced drivers. In Systems, Man and Cybernetics, 2008. SMC 2008. IEEE International Conference on October, pp. 804-809

[21] Morioka, M., \& Griffin, M. J., 2009. Equivalent comfort contours for vertical vibration of steering wheels: Effect of vibration magnitude, grip force, and hand position. Applied ergonomics, 40(5), pp. 817-825.

[22] International Standards Office, 2001. ISO 13091-1, Mechanical vibrations. Vibrotactile perception thresholds for the assessment of nerve dysfunction. Methods of measurement at the fingertips, London: BSI

[23] Giacomin, J., \& Abrahams, O., 2000. Human fatigue due to automobile steering wheel vibration. In SIA Conference on Car and Train Comfort, Le Mans, France, 15th to 16th November.

[24] Giacomin, J. and Screti, A., 2005. Upper body discomfort due to driving: effect of driving experience, gender and vehicle age, Zeitschrift Arbeitswissenschaft, 5, pp. 409-418.

[25] British Standards Institution, 1993. BS 6840-2: 1993 Sound system equipment: glossary of general terms and calculation methods. London: BSI.

[26] Fujikawa, K., 1998. Analysis of steering column vibration. Motion \& Control, 4(1), pp. 37-41.

[27] Pak, C. H., Lee, U. S., Hong, S. C., Song, S. K., Kim, J. H., \& Kim, K. S., 1991. A study on the tangential vibration of the steering wheel of passenger car (No. 912565). SAE Technical Paper.

[28] Kulkarni, K. B., \& Thyagarajan, R. S., 2001. Optimizing the effects of body attachment stiffness on steering column in-vehicle modes (No. 2001-01-0041). SAE Technical Paper. 